\title{
The heat sensitive factor (HSF) of Yersinia ruckeri is produced by an alkyl sulphatase involved in sodium dodecyl sulphate (SDS) degradation but not in virulence
}

Roberto Navais, Jessica Méndez, Desirée Cascales, Pilar Reimundo and José A Guijarro*

\begin{abstract}
Background: The heat sensitive factor (HSF) of the fish pathogen Yersinia ruckeri was previously identified as an unusual band on SDS-PAGE. According to this, Y. ruckeri strains were classified in $\mathrm{HSF}^{+}$and $\mathrm{HSF}^{-}$in terms of the presence/absence of the factor. Experiments carried out by injection challenge with $\mathrm{HSF}^{+}$strains caused high mortalities in rainbow trout. In contrast, $\mathrm{HSF}^{-}$strains did not cause mortality. In conclusion, HSF appeared to be a relevant virulence factor in Y. ruckeri.

Results: We report here the identification and study of the gene coding for the enzyme involved in the production of HSF. Culture medium containing SDS and Coomassie brilliant blue dye was used to screen a mini-Tn5 Km2 mutant library of $Y$. ruckeri 150. Blue colonies lacking a surrounding creamy deposit, a phenotype described in former studies as $\mathrm{HSF}^{-}$, were identified. DNA sequence analysis of a selected mutant revealed that this had a transposon interruption in a chromosome-located gene which codes for a heat sensitive alkyl sulphatase of $78.7 \mathrm{kDa}$ (YraS; Yersinia ruckeri alkyl sulphatase) which is able to degrade SDS to 1-dodecanol. As it was expected, the introduction of the yraS gene into an $\mathrm{HSF}^{-}$strain turned this into $\mathrm{HSF}^{+}$. Surprisingly, although the protein allows Y. ruckeri to degrade SDS, the bacterium could not use this compound as the sole carbon source. Moreover, the yraS mutant showed a similar level of SDS resistance to the parental strain. It was the interruption of the acrA gene which made $Y$. ruckeri sensitive to this compound. $\mathrm{LD}_{50}$ experiments showed a similar virulence of the yraS mutant and parental strain.

Conclusions: The HSF of Y. ruckeri is the product of the alkyl sulphatase YraS, able to degrade SDS to 1-dodecanol. This degradation is not linked to the utilization of SDS as a carbon source and surprisingly, the enzyme is not involved in bacterial virulence or in the high SDS resistance displayed by the bacterium. This role is played by the AcrAB-TolC system.
\end{abstract}

Keywords: Yersinia ruckeri, HSF factor, Alkyl sulphatase, Virulence

\section{Background}

Yersinia ruckeri is a Gram-negative bacterium alternating between planktonic and host interaction states. It is the causative agent of enteric redmouth disease (ERM) affecting mainly salmonids in fish farms, which leads to important economic losses in aquaculture worldwide. Four serological groups [1] and two biotypes [2] of $Y$. ruckeri are currently proposed. Amongst them,

\footnotetext{
*Correspondence: jaga@uniovi.es

Área de Microbiología, Departamento de Biología Funcional, Facultad de Medicina, IUBA, Universidad de Oviedo, 33006 Oviedo, Asturias, Spain
}

serotype 1 is the most virulent, being commonly isolated from outbreaks in fish farms. Whereas the mechanisms involved in the virulence of human pathogenic Yersinia species have been studied in depth, only a few pathogenic mechanisms of $Y$. ruckeri have been described [3-8]. In this context, of special interest is the work of Furones et al. [9], who found an association between the virulence of $Y$. ruckeri serotype I strains and the presence of a heatsensitive factor (HSF), identified as a sodium dodecyl sulphate polyacrylamide gel electrophoresis (SDS-PAGE) band of approximately $120 \mathrm{kDa}$ from cell extracts. The 
strains were classified in $\mathrm{HSF}^{+}$and $\mathrm{HSF}^{-}$in terms of the presence/absence of the factor [9]. Experiments carried out by injection challenge with $\mathrm{HSF}^{+}$strains caused high mortalities in rainbow trout. In contrast, $\mathrm{HSF}^{-}$strains did not cause mortality. These authors concluded that HSF appeared to be a relevant virulence factor in $Y$. ruckeri. In order to be able to distinguish $\mathrm{HSF}^{+}$and $\mathrm{HSF}^{-}$strains routinely, Furones et al. [10] developed a differential culture medium containing SDS and the Coomassie brilliant blue dye. In this medium, the colonies of the $\mathrm{HSF}^{+}$strains appeared as white, due to a creamy deposit around the colony, whereas those of $\mathrm{HSF}^{-}$strains did not form this deposit and became deeply blue.

Given the relationship between the presence of the HSF and the virulence of $Y$. ruckeri, we decided it would be interesting to investigate the gene coding for this factor. The HSF was found to be produced by an alkyl sulphatase, a protein which is not related to the virulence of the bacterium, but with potential usefulness for removing surfactant from the environment.

\section{Results}

Creamy white $Y$. ruckeri colony phenotype $\left(\mathrm{HSF}^{+}\right)$growing in the presence of SDS is caused by an alkyl sulphatase (YraS)

A mini-Tn5 Km2 Y. ruckeri transposon library was screened for colonies lacking the creamy white deposit around the colonies on TSA medium containing SDS and Coomassie brilliant blue (Figure 1). Southern blot and sequence analysis of the interrupted genes in several blue coloured colonies showed that all of them presented a unique chromosomal transposon insertion in a 2,127 bp open reading frame. Putative -10 (ATTATT) and -35 (ATAACA) promoter sequences, and ribosome-binding site (AAGGA) were identified upstream of the ORF, and a stem-loop palindromic sequence corresponding to a rhoindependent terminator was located at the 3 'end of the gene (Figure 2).

The product of the interrupted gene, a protein of 708 amino acids, shows a high degree of identity with other proteins defined as alkyl sulphatases or SDS hydrolases from different bacteria such as Microcystis aeruginosa (71\%) (CCI04676), Thiocystis violascens (63\%) (YP_006413521), Vibrio cholerae (52\%) (EGS71352) and Pseudomonas aeruginosa (51\%) [11].

In silico analysis indicated that the protein carries an $\mathrm{N}$-terminal signal peptide of 24 amino acid residues and contains two domains: the cl00446, characteristic of the metalo- $\beta$-lactamase family, and the COG2015, typical of alkyl sulphatases (Figure 2). Both domains are present in the SdsA and SdsA1 alkyl sulphatases from Pseudomonas sp. ATCC19151 (AAA25989) [12] and P. aeruginosa PAO1 (NP_249431) [11], respectively. Additionally, the protein harbours, at its $\mathrm{N}$-terminal sequence, a $\mathrm{Zn}^{2+}$-binding motif (THxHxDHxGG-102-E-18-AE44-H) characteristic of metallo- $\beta$-lactamase-related enzymes, also present in the SdsA and SdsA1 alkyl sulphatases [11,12] (Figure 2).

The in silico results were in concordance with data obtained from the analysis by gas chromatography-mass spectrometry of the creamy-white compound produced by cultures of the $Y$. ruckeri parental strain grown in NB with $0.25 \%$ SDS. The compound was identified as 1-dodecanol (Additional file 1: Figure S1), the molecule resulting from the hydrolysis of SDS by an alkyl sulphatase [13].

The phylogenetic tree, based on the YraS protein of different microorganisms, showed that $Y$. ruckeri does not share any evolutionary origin with the bacteria harbouring
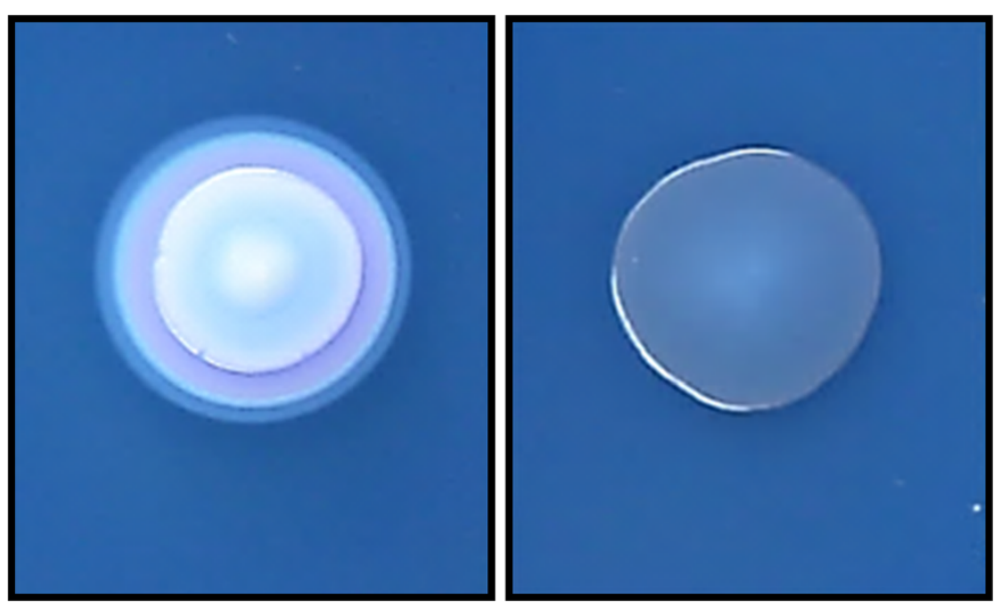

Figure 1 Colony morphology of $Y$. ruckeri strains grown on TSA-SDS containing Coomassie brilliant blue dye showing the $\mathrm{HSF}^{+}(Y$. ruckeri 150 parental strain, left) and $\mathrm{HSF}^{-}$(mutant obtained after the screening of a Y. ruckeri $150 \mathrm{Tn} 5 \mathrm{Km} 2$ library, right) phenotypes. Aliquots of $5 \mathrm{\mu l}$ from early stationary phase cultures of the parental and mutant strains were spotted onto TSA-SDS Coomassie brilliant blue medium. After $48 \mathrm{~h}$ of incubation at $28^{\circ} \mathrm{C}$ colonies were photographed. Creamy white colony $\left(\mathrm{HSF}^{+}\right)$contrasts with the blue colony $\left(\mathrm{HSF}^{-}\right)$phenotype. 


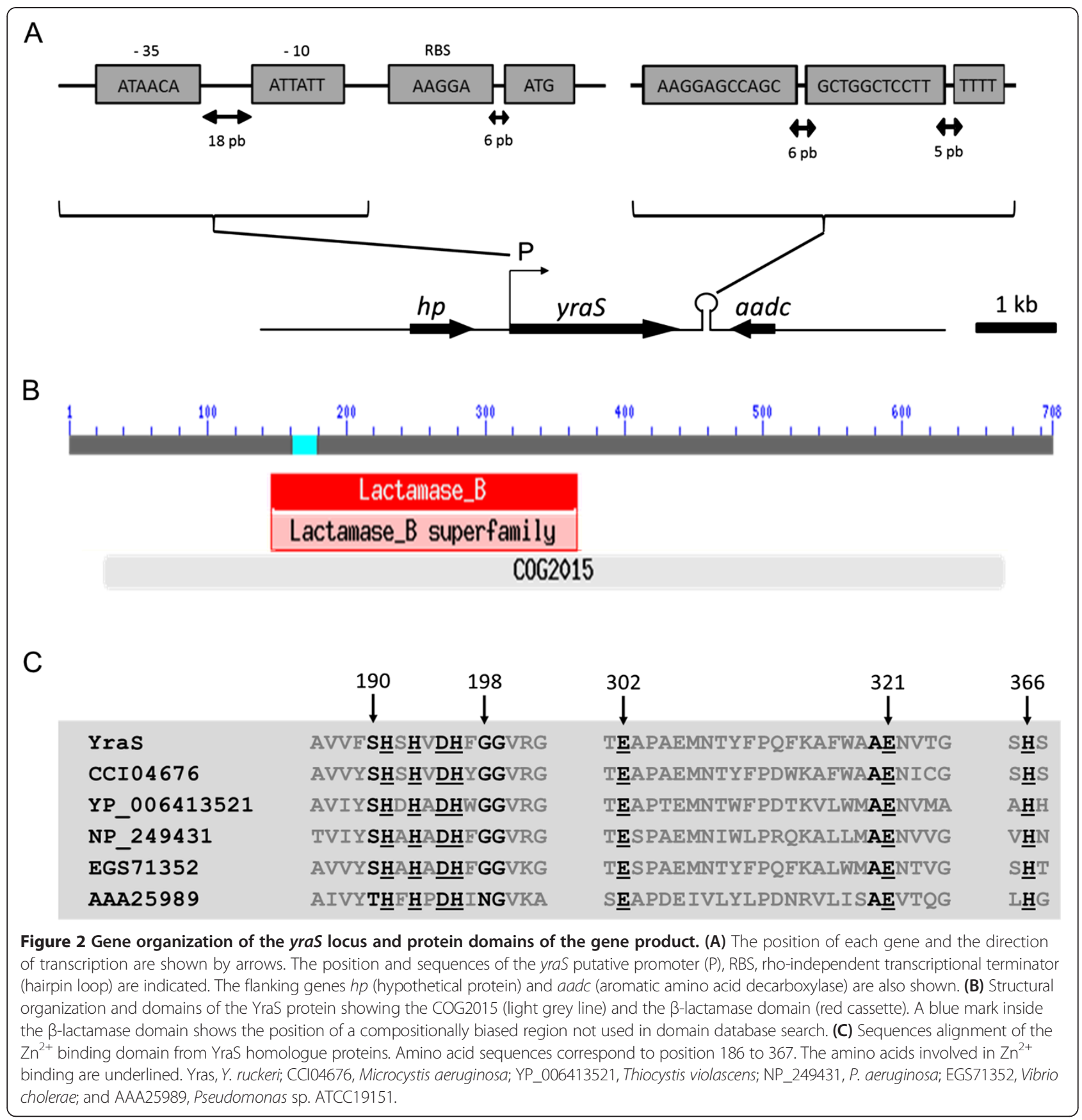

an YraS-homologous protein (Additional file 2: Figure S2). However, all the bacteria that are closer to $Y$. ruckeri in the phylogenetic tree, with the exception of Sinorhizobium meliloti, are also aquatic. One example is Photobacterium profundum, a psychrophilic marine bacterium belonging to the Vibrionaceae family. Proteins homologous to YraS were also found in other Enterobacteriaceae species such as Klebsiella oxytoca, Klebsiella variicola and Salmonella enterica. However, the proteins of these bacteria are very distant in the phylogenetic tree from the YraS protein of Y. ruckeri (Additional file 2: Figure S2).
YraS is the HSF factor

In order to go further into the relationship between the yraS gene and the $\mathrm{HSF}^{+}$phenotype, defined by a creamy deposit around the colony on SDS containing media (Figure 3A), one of the obtained mutants showing an $\mathrm{HSF}^{-}$colony phenotype ( $\mathrm{HSF}^{-1}$; Figure $3 \mathrm{~B}$ ) was complemented with the yraS gene. As can be observed in Figure $3 \mathrm{C}$, a complete reversion of the $\mathrm{HSF}^{-}$to $\mathrm{HSF}^{+}$colony phenotype was achieved. Furthermore, when the yraS gene was introduced in $Y$. ruckeri 956, a serotype II strain showing an $\mathrm{HSF}^{-}$phenotype, the colony phenotype changed to 


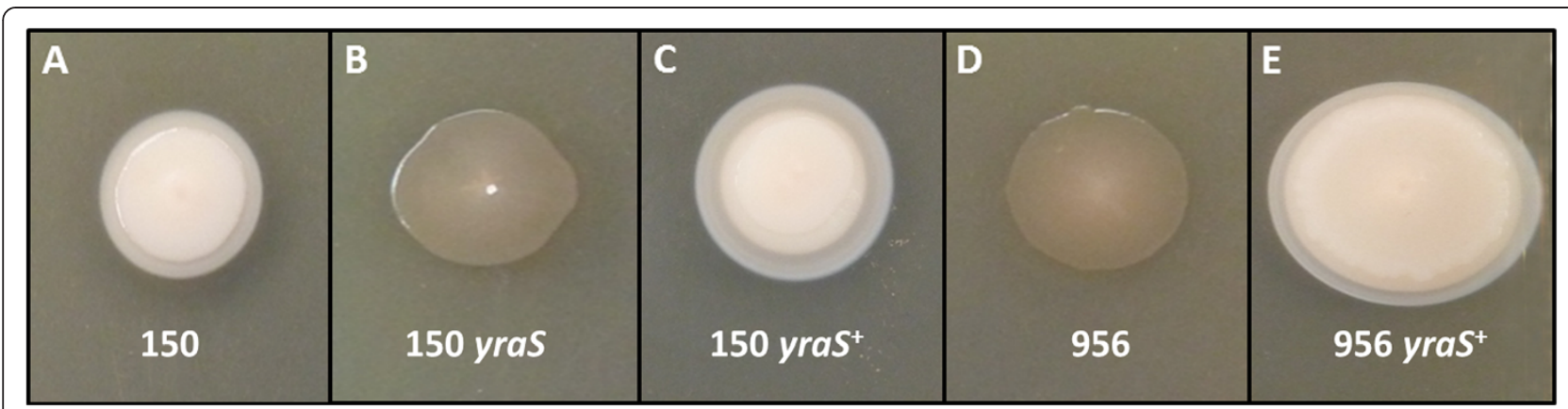

Figure 3 Colony morphology of different $Y$. ruckeri strains grown on TSA-SDS showing the $\mathrm{HSF}^{+}$and $\mathrm{HSF}^{-}$phenotypes. Strains were point inoculated and after $120 \mathrm{~h}$ at $28^{\circ} \mathrm{C}$ colonies were photographed. A. Y. ruckeri 150 parental strain. The HSF ${ }^{-}$phenotype of yraS mutant $\left(\mathrm{HSF}^{-1}\right) \mathbf{( B )}$, and 956 strains (D) changes to $\mathrm{HSF}^{+}$phenotype (C and $\mathbf{E}$, respectively), when they are complemented by the yraS gene.

$\mathrm{HSF}^{+}$(Figure 3D,E). Both, yraS mutant and 956 strains, when complemented with the yraS gene, originated a larger creamy deposit than that of the parental strain, probably owing to gene dosage effect (Figure 3C,E). In conclusion, the yraS gene is responsible for the $\mathrm{HSF}^{+}$phenotype of $Y$. ruckeri colonies on SDS-containing media.

To confirm that the YraS protein was linked to the HSF factor, defined by Furones et al. [9] as a $120 \mathrm{kDa}$ band in SDS-PAGE gels, cell extracts from the different $Y$. ruckeri parental, yraS mutant, 956 and complemented strains were obtained and separated by SDS-PAGE under the conditions previously described [9]. Figure 4A, lane 1 , shows the presence in the $Y$. ruckeri parental strain of a band, resulting from the degradation of the
SDS present in the gel, with an apparent molecular mass of approximately $120 \mathrm{kDa}$. This band disappeared in the yraS mutant (Figure 4A, lane 2) and returned when the mutant was complemented with the yraS gene (Figure 4A, lane 3). This band was also absent in the $\operatorname{HSF}^{-}$Y. ruckeri 965 strain (Figure 4B, lane 1), and again appeared when this strain was yraS complemented (Figure 4B, lane 2). The presence in the gel of this $120 \mathrm{kDa}$ band was temperature-dependent. Indeed, when the cell extracts of the $\mathrm{HSF}^{+}$strains were heated to $100^{\circ} \mathrm{C}$ for $10 \mathrm{~min}$, before SDS-PAGE separation, the $120 \mathrm{kDa}$ band was absent (Figure 4A, lane 4). Moreover, when the gel was stained with Sudan black dye the $120 \mathrm{kDa}$ band became stained (Additional file 3: Figure S3). Both types of behaviour were defined previously for the HSF factor [9].
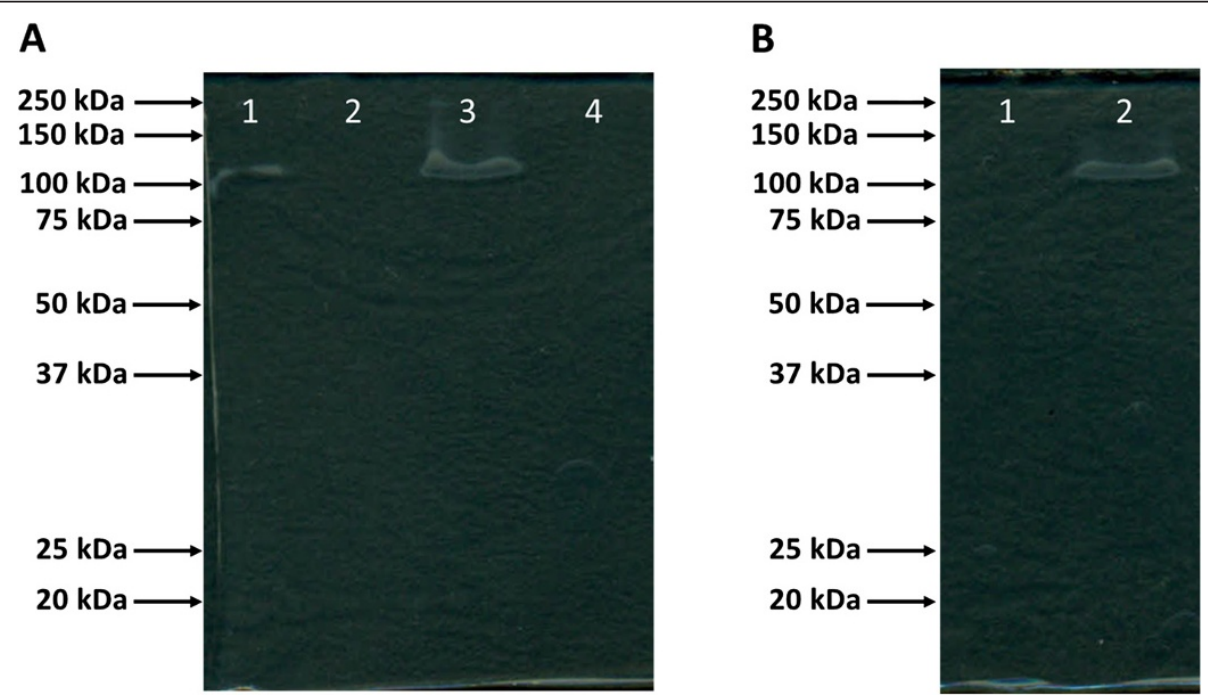

Figure 4 Zymograms showing SDS hydrolysis after SDS-PAGE of crude extract of different $Y$. ruckeri strains. SDS-PAGE of bacterial cell extracts was performed at $15 \mathrm{~mA}$ in a cool room for $16 \mathrm{~h}$. Then, gels were incubated at $20^{\circ} \mathrm{C}$ for $4 \mathrm{~h}$ and kept 1 additional hour at $4^{\circ} \mathrm{C}$ for SDS precipitation. Bands of SDS hydrolysis activity appear as clear zones against an opaque gel. (A) Cell extract from: lane 1, Y. ruckeri (parental strain); lane 2, yraS ; lane 3, yraS ; lane 4, parental strain heated at $100^{\circ} \mathrm{C}$ for $10 \mathrm{~min}$. (B) Cell extract from: lane 1, Y. ruckeri 956; lane 2, 956yraS ${ }^{+}$. Molecular mass markers (in $\mathrm{kDa}$ ) are indicated on the left side of each gel. Photographs were taken on a dark background to contrast the bands. There was a match between the appearance in the SDS-PAGE of the $120 \mathrm{kDa}$ SDS hydrolysis bands and the presence of the yraS gene in the strains. 


\section{$\mathrm{HSF}^{+} / \mathrm{HSF}^{-}$phenotype correlates with the presence/ absence of the yraS gene in $Y$. ruckeri strains}

A set of $Y$. ruckeri strains, including some used in the study of Furones et al. $[9,10]$, were analysed by PCR for the presence of the yraS gene and also tested for colony morphology on SDS-containing medium. In all the cases, there was a match between colony morphology and the presence of the yraS gene: all the $\mathrm{HSF}^{+}$strains harboured the yraS gene and presented a white creamy deposit surrounding the colonies, whereas in $\mathrm{HSF}^{-}$strains the yraS gene was absent and colonies lacked the white creamy deposit (Figure 5).

\section{The yraS gene is not involved in virulence and it has no nutritional role}

The presence in Y. ruckeri of the HSF factor, now defined as the product of the YraS enzyme, was previously associated with virulence $[9,10]$. In order to define the role of this factor in virulence, $\mathrm{LD}_{50}$ experiments were carried out in groups of 10 rainbow trout fish (weight 10-15 g) intraperitoneally injected with $100 \mu \mathrm{l}$ of parental and yraS mutant strains dilutions from $10^{2}$ to $10^{8} \mathrm{CFU} / \mathrm{ml}$. Fish death occurred along the 7 days period and cumulative mortality curves were similar for both strains in each dilution (Additional file 4: Figure S4). At the end of this period, $\mathrm{LD}_{50}$ values obtained were $1.0 \times 10^{2} \mathrm{CFU}$ (with lower and upper $95 \%$ confidence limits of $1.3 \times 10$ and $4.0 \times$ $10^{2} \mathrm{CFU}$ ), and $1.1 \times 10^{2} \mathrm{CFU}$ (with lower and upper $95 \%$ confidence limits of 9.0 and $\left.4.8 \times 10^{2} \mathrm{CFU}\right)$, respectively. These results showed that the YraS protein is not a virulence factor.

Constitutive expression of the yraS gene seems to occur, since the YraS protein is present in both NB and M9C media. A first approach to the role of the YraS protein was inferred from experiments in which glucose was added to the M9C medium containing $1 \%(\mathrm{w} / \mathrm{v})$ SDS. After $96 \mathrm{~h}$ of incubation at $28^{\circ} \mathrm{C}$, no degradation at all of SDS by the parental strain occurred when glucose was present in the culture medium. In the same way, the parental strain showed an $\mathrm{HSF}^{-}$phenotype when glucose was present in M9C medium containing SDS (Additional file 5: Figure S5). This result could be a consequence of a

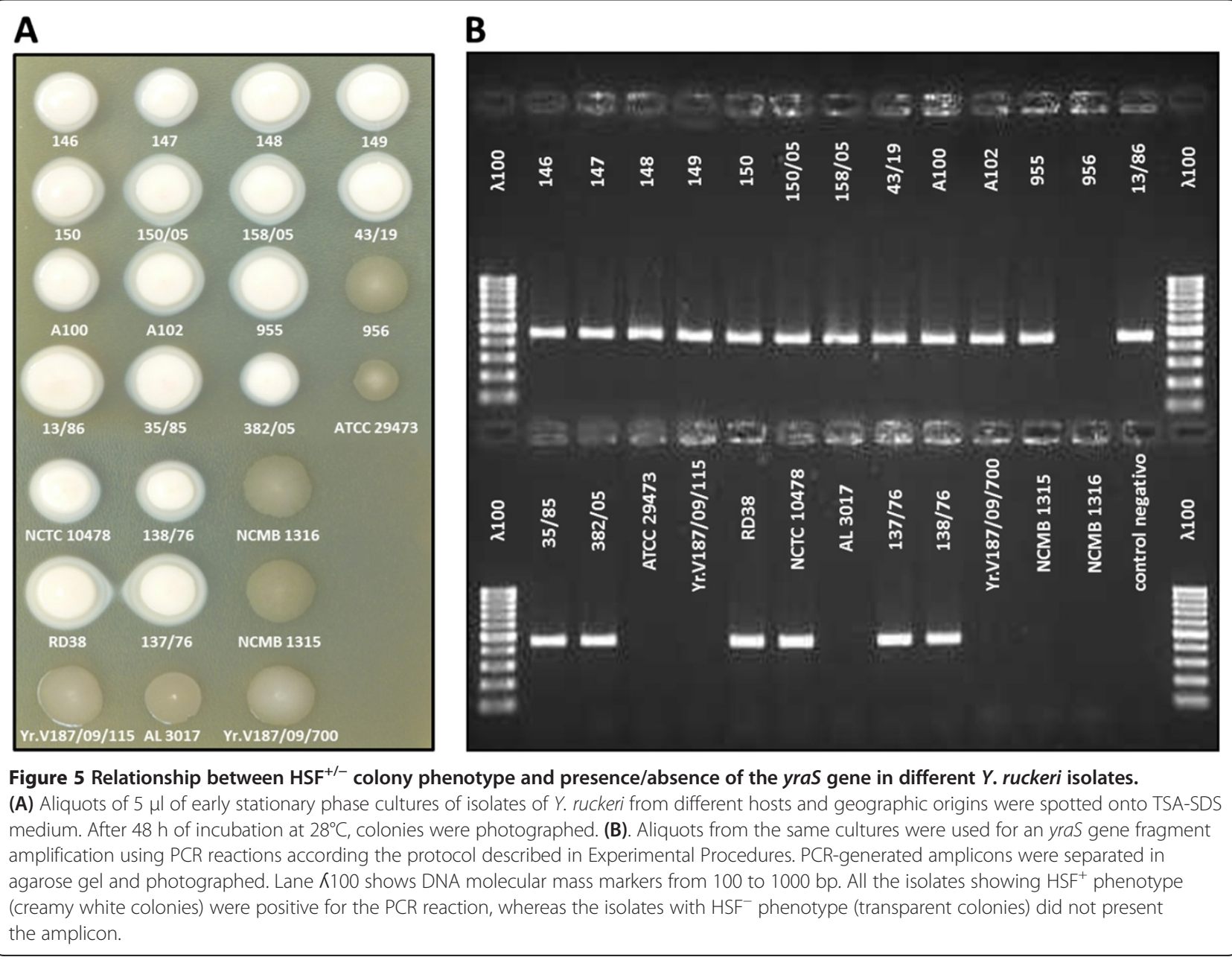


catabolic repression mechanism involved in the regulation of the yraS gene. Therefore, it would be possible that $Y$. ruckeri uses SDS as an additional carbon source for bacterial growth. However, when the different Y. ruckeri strains were incubated in M9 solid and liquid media containing different amounts of SDS as sole carbon source no growth at all was observed. Definitely, $Y$. ruckeri SDS degradation by YraS is not linked to the utilization of this detergent as a sole carbon source. Moreover, the presence in the culture media of different amounts of sulphate did not produce any visible alteration in SDS degradation, suggesting that sulphate is not involved in the regulation of the yraS gene.

The protein YraS is not involved in SDS-resistance; instead, this property lies within the AcrAB-Tolc system Growth of the yraS mutant strain in NB medium containing $0.25 \%$ SDS at $28^{\circ} \mathrm{C}$ was similar to that of the parental strain, although a greater decrease in cell viability occurred in the mutant strain during the stationary phase of growth (Figure 6). SDS was totally degraded by the parental strain after $24 \mathrm{~h}$ of incubation at $28^{\circ} \mathrm{C}$, but no degradation at all occurred during the growth of the yraS mutant (Figure 6). Percentages of $0.5 \%$ and $1 \%$ SDS were not totally degraded by the parental strain, probably because nutrient depletion occurred before all the detergent was hydrolysed.

To examine the role of the YraS protein in the physiology of the bacterium, the MIC of SDS for different $Y$. ruckeri strains was determined. The results indicated that an identical level of resistance to SDS $(\mathrm{MIC}=3.2 \% \mathrm{w} / \mathrm{v})$ was found amongst $Y$. ruckeri parental, yraS mutant and complemented $\mathrm{yraS}^{+}$strains. In addition, no major resistance to SDS was found when the $Y$. ruckeri 956 strain was complemented with the yraS gene, the MIC for both strains was $0.8 \%(\mathrm{w} / \mathrm{v})$. These results indicated that the alkyl sulphatase is not a relevant SDSresistance mechanism in the bacterium. Similarly, both parental and mutant strains presented the same MIC for Triton X-100, Tween 80 and bile salts (data not shown).

In order to elucidate the system responsible for the high SDS resistance of the bacterium, a mini-Tn5 Km2 mutant library of $Y$. ruckeri was screened to select a mutant unable to grow in the presence of $0.5 \%$ SDS. The gene whose interruption was responsible for that phenotype codes for a protein homologous to AcrA, one of the components of the AcrAB-TolC system. This is involved in pumping out of the cell a wide variety of

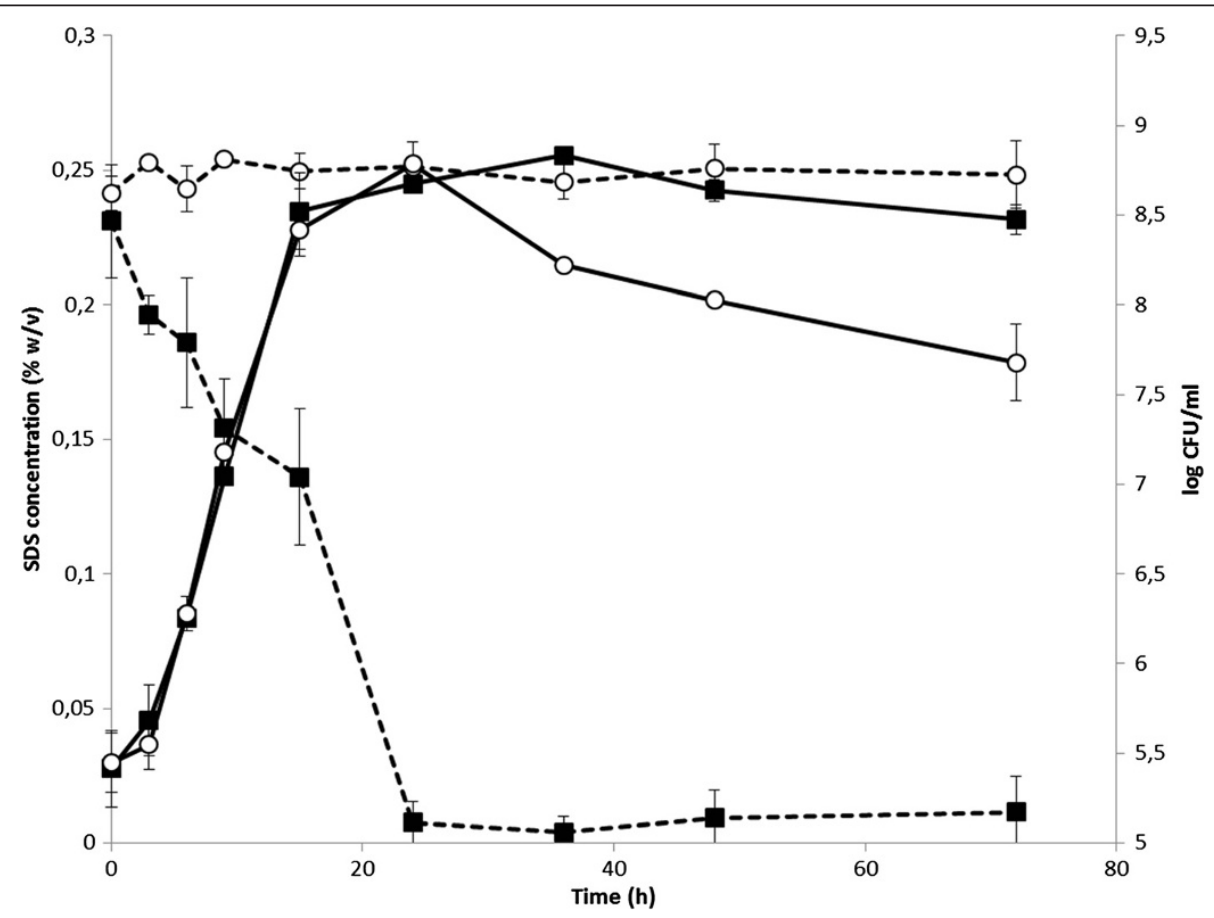

Figure 6 Growth curves and SDS degradation by Y. ruckeri parental and yraS mutant strains. Cultures were incubated in NB containing $0.25 \%(\mathrm{~W} / \mathrm{V}) \mathrm{SDS}$ at $28^{\circ} \mathrm{C}$ and $250 \mathrm{rpm}$ and, at different times, samples were withdrawn and growth determined by plate counting. Simultaneously, the presence of SDS in the culture supernatant was quantified by the Stains-all method [14]. Continuous line and dotted line represents the growth and SDS degradation of parental (- and yraS ${ }^{-}$(o) strains. Data represent the mean \pm standard deviation of three independent experiments. Growth was similar for the two strains, but SDS was completely degraded by the parental strain after $24 \mathrm{~h}$ of incubation, whereas no degradation at all occurred by the yraS mutant strain. 
compounds including detergents and antibiotics in bacteria such as Escherichia coli and Salmonella enterica [15-17]. Phenotypic analysis of this mutant showed that it was sensitive to SDS, having a MIC of $0.00625 \% \mathrm{w} / \mathrm{v}$, instead of the $3.2 \% \mathrm{w} / \mathrm{v}$ of the parental strain. Additionally, the acr $A$ mutant was significantly more sensitive than the parental strain to Triton X-100, bile salts and the antimicrobials tetracycline, oxitetracycline, ciprofloxacin, chloramphenicol and oxolinic acid (data not shown).

\section{Discussion}

Virulence in $Y$. ruckeri was previously correlated with the presence of the $\mathrm{HSF}^{+}$colony phenotype [9]. In this work, this $\mathrm{HSF}^{+}$phenotype was linked to the product of the $y r a S$ gene which corresponds to an alkyl sulphatase. This enzyme is involved in the degradation of alkyl sulphate esters, such as SDS, generating as a first product water insoluble 1-dodecanol. This compound, which, in $Y$. ruckeri is accumulated in the medium, forms a white creamy deposit around colonies and leads to a band of $120 \mathrm{kDa}$ in SDS-PAGE gels. Both phenotypes were previously observed by Furones et al., [9,10]. According to in silico analysis, the YraS enzyme falls into a new class of alkyl sulphatases characterized by the presence of a metallo- $\beta$-lactamase domain and found mostly in gram-negative bacteria [11]. This kind of enzymes cleaves alkyl sulphates such as SDS into the corresponding alcohol.

The YraS activity was identified in SDS-PAGE as a diffuse SDS-hydrolytic band of $120 \mathrm{kDa}$, approximately. This result is coincident with the molecular mass of HSF described by Furones et al. [9]. According to this, and taking into account that the molecular mass of the YraS, deduced from the amino acid sequence, was $78.7 \mathrm{kDa}$, the active form of the enzyme should correspond to a protein dimer structure. This also agrees with the dimeric structure of the active form of the SdsA1 from $P$. aeruginosa, which provides resistance to high concentrations of SDS [11]. In the same way, a lipid core structure for HSF was suggested, since the $120 \mathrm{kDa}$ band was stained with Sudan black, a dye used for lipid detection [9]. Our results showed that the $120 \mathrm{kDa}$ band was also stained with this dye. Therefore, the lipidic core structure of HSF suggested by Furones et al. [9] was correct, since it is likely that the compound stained with Sudan black is the fatty alcohol 1-dodecanol, the product of the alkyl sulphatase action on SDS. Nevertheless, we must link the HSF to the alkyl sulphatase rather than to the product of SDS degradation by the enzyme. This fact is consistent with the absence of the band when the cell extract was heat-inactivated before electrophoresis. Comparative analysis of parental and yraS mutant $Y$. ruckeri strains, complementation studies, as well as PCR detection of the yraS gene in different strains of $Y$. ruckeri, confirm that YraS is responsible for the production of the HSF factor. These results, together with the presence of a single $120 \mathrm{kDa}$ band in the cell extracts of the parental $Y$. ruckeri strain, strongly suggested that, under these experimental conditions, there were no additional alkyl sulphatases involved in the degradation of SDS, and the YraS protein was the only one responsible for this activity. This differs from different reports that indicated that bacteria able to degrade alkyl sulphate esters possess multiple sulphatase enzymes [11,18-22].

Our results indicate that there was no relation between the presence of HSF factor and virulence in $Y$. ruckeri. Indeed, results of the rainbow trout infection experiments carried out with the parental and yraS mutant strains, showed that both strains behaved similarly as virulent bacteria. These results are not incompatible with the one obtained by Furones et al., $[9,10]$ because although YraS is not a virulence factor its presence could be linked to the existence in the bacterium of specific virulence factors. In this way, YraS could be a hallmark of virulence even though it is not involved in pathogenesis. This could explain why $\mathrm{HSF}^{+}$strains are always virulent $[9,10]$.

The YraS protein was produced by the bacterium when growing in media lacking SDS and therefore, it seems that the gene is constitutively expressed. However, YraS activity was absent when the bacterium was grown in the presence of glucose, suggesting a regulation of the yraS gene by catabolic repression. Nevertheless, the absence of bacterial growth in the presence of SDS as a sole carbon source as well as the accumulation of 1-docecanol in the culture media, indicate that $Y$. ruckeri, in contrast to $P$. aeruginosa [11], is unable to use this detergent as sole carbon source. 1-dodecanol, resulting from SDS degradation by the YraS protein, is likely to enter the bacterium where it would be oxidized to 1-dodecanoic acid by the action of the appropriate alcohol dehydrogenase and aldehyde dehydrogenase to be assimilated as a carbon source. Therefore, it seems that the absence of one or more of these systems could be a problem which prevents further metabolic assimilation of 1-dodecanol by $Y$. ruckeri.

No alteration in detergent hydrolysis by $Y$. ruckeri was observed when inorganic sulphate was added to the media. Our results suggest that in $Y$. ruckeri this enzyme is not involved in the sulphur cycle and is not a system for scavenging sulphate from the environment. Whereas bacterial aryl sulphatases have been involved in sulphate scavenging [23], alkyl sulphatases seem to have additional roles $[11,24,25]$. In conclusion, and according to the results, it seems that the $y \mathrm{raS}$ gene is not related to carbon or sulphur utilization.

There are many reports of bacteria able to degrade different amounts of SDS. For example, a P. putida SP3 strain was able to completely degrade $0.1 \%$ SDS in $16 \mathrm{~h}$ [26]. However, most of the SDS-degrading bacteria 
isolated presented lower rates, needing 4 to 10 days of incubation to completely degrade percentages of 0.05 to $0.2 \%$ of SDS $[27,28]$. Interestingly, $Y$. ruckeri degrades $0.25 \%$ SDS in NB within $24 \mathrm{~h}$ of incubation at $28^{\circ} \mathrm{C}$, and can grow in the presence of up to $1.6 \%$ of this detergent. Introduction of the yraS gene in $Y$. ruckeri strains lacking the capacity to degrade SDS enables these strains to do so. However, Y. ruckeri SDS resistance is not yraSdependent since the growth curve of the yraS mutant strain in the presence of $0.25 \%$ SDS was similar to that of the parental strain. To our knowledge, it is here established for the first time that a gene involved in SDS degradation is not associated with the resistance of the bacterium to this detergent. Usually it is assumed that the presence of alkyl sulphatases in a particular bacterium is related to its resistance to toxic alkyl sulphate molecules. However, in fact, resistance of Y. ruckeri to SDS lies in the AcrAB-TolC system, a pump involved in the excretion of multiple compounds such as antimicrobials and detergents in different bacteria [15-17], as was indicated by the analysis of an SDS-sensitive Y. ruckeri acrA mutant. Therefore, in this particular case, and probably in other bacteria bearing this kind of sulphatasecoding gene, it may be that the yraS gene is only present in naturally SDS resistant $Y$. ruckeri strains. These characteristics, high SDS resistance and degradation capacity, make this bacterium potentially useful as a tool for removing anionic surfactants from different sources.

It is interesting to speculate about the presence of this enzyme in a bacterium alternating between planktonic and host interaction states. The results indicate that the protein does not have a significant role in the infectious process or in nutrition or detergent resistance. However, the constitutive expression in nutritionally rich and poor media suggests that the protein provides an advantage to the bacterium in its natural environment. The high resistance to SDS depending on the AcrAB-TolC system, together with the high rate of degradation of this compound by the alkyl sulphatase YraS, reinforces the potential usefulness of bacteria possessing high expression systems of both mechanisms in the fight against surfactant pollution in bioremediation.

\section{Conclusions}

We have showed in this work the nature of the previously defined HSF factor of $Y$. ruckeri as the product of SDS degradation by the YraS alkyl sulphatase. Although in former works it was established a correlation between the possession of HSF and the virulence of this microorganism, our results indicate that the factor is not necessary for bacterial pathogenicity. YraS was able to degrade SDS but it does not contribute, at least in a relevant way, to the high SDS resistance observed in this bacterium. This function corresponds to the AcrAB-TolC system.

\section{Methods}

\section{Bacterial strains and culture conditions}

The bacterial strains and plasmids used in this study are listed in Tables 1 and 2, respectively. E. coli strains were routinely grown in $2 \mathrm{xTY}$ broth and agar, and $Y$. ruckeri strains in nutrient broth (NB) and nutrient agar (NA). Screening of $Y$. ruckeri strains for the $\mathrm{HSF}^{+} / \mathrm{HSF}^{-}$colony phenotype was performed on TSA medium supplemented with 1\% SDS (TSA-SDS). Coomassie brillant blue dye was added to molten TSA-SDS medium to provide final concentrations of $100 \mu \mathrm{g} / \mathrm{ml}$, as described by Furones et al., [10]. Experiments on SDS as a nutrient were performed using the minimal medium described by Romalde et al. [29] without glucose (M9) and M9C (M9 containing $2 \mathrm{~g} / \mathrm{l}$ casamino acids) broth and agar media supplemented with different percentages of SDS. When it was required glucose was added to M9C to a concentration of $0.5 \%(\mathrm{w} / \mathrm{v})$. For motility experiments, a semisolid tryptone agar medium (5 g/l NaCl, $10 \mathrm{~g} / \mathrm{l}$ tryptone, $0.6 \% \mathrm{w} / \mathrm{v}$ agar) was used. Liquid cultures were incubated at $37^{\circ} \mathrm{C}$ for $E$. coli and $18^{\circ} \mathrm{C}$ and $28^{\circ} \mathrm{C}$ for $Y$. ruckeri in orbital shakers at $250 \mathrm{rpm}$. Growth was monitored by determining the $\mathrm{OD}_{600}$. When SDS was present in the liquid culture growth was determined by serial dilutions and plate counts. When required, the following compounds were added to the media: $100 \mu \mathrm{g} / \mathrm{ml}$ ampicillin, $0.1 \mu \mathrm{g} / \mathrm{ml}$ cephotaxime, $50 \mu \mathrm{g} / \mathrm{ml}$ kanamycin or streptomycin.

\section{Mutant selection, DNA sequencing and mutant complementation}

A mini-Tn5 Km2 transposon based Y. ruckeri $150 \mathrm{mu}-$ tants library was generated by using the pUT mini-Tn5 $\mathrm{Km} 2$ plasmid. This plasmid was transferred by conjugation from E. coli S17 K1pir to Y. ruckeri 150 strain [34]. Transconjugants were first selected on NA plates supplemented with kanamycin and cephotaxime by incubation for two days at $28^{\circ} \mathrm{C}$. Approximately 10000 transposon mutants were replicated onto TSA-SDS medium containing Coomassie brillant blue dye and incubated for five days. Colonies showing an $\mathrm{HSF}^{-}$phenotype characterized by a deep blue colour were selected. For testing that selected transconjugants presented a sole transposon insertion, selected colonies were analysed by Southern blotting using a DIG DNA detection kit (Roche) following the protocol described by the manufacturer. Total DNA from transconjugants and the parental strain was isolated (GenElute Mammalian Genomic DNA Purification Kit-Sigma) and digested with EcoRI and XbaI restriction enzymes. Then, DNA fragments were subjected to $0.75 \% \mathrm{w} / \mathrm{v}$ agarose gel electrophoresis at $100 \mathrm{~V}$ for $2 \mathrm{~h}$, transferred to a nylon membrane (Amersham Biosciences) and fixed with UV irradiation. The labelled region of the kanamycin gene from the Tn5 transposon was used as a 
Table 1 Bacterial strains used in this study

\begin{tabular}{|c|c|c|c|c|}
\hline Strain & Serotype & HSF & Other characteristics & Source of reference \\
\hline \multicolumn{5}{|l|}{ Y. ruckeri } \\
\hline 150 & I & + & Isolated during outbreaks of ERM disease in Denmark & J.L. Larsen, University of Frederiksberg (Denmark) \\
\hline 150 acrA & I & + & acrA::-mini-Tn5 Km2 Kmr & This study \\
\hline 150 yras & 1 & - & yraS::mini Tn5 Km2 Kmr & This study \\
\hline 150 yras+ & I & + & 150 yraS harboring pGBM5-yraS & This study \\
\hline $146,147,148,149$ & 1 & + & Isolated during outbreaks of ERM disease in Denmark & J.L. Larsen, University of Frederiksberg (Denmark) \\
\hline 955 & I & + & Trout-isolated strain & CECT (Spanish Type Culture Collection) \\
\hline 956 & $\|$ & - & Trout-isolated strain & CECT (Spanish Type Culture Collection) \\
\hline $43 / 19$ & I & - & Trout-isolated strain & CECT (Spanish Type Culture Collection) \\
\hline 956 yraS+ & $\|$ & + & 956 harboring pGBM5-yraS & This study \\
\hline $35 / 85^{*}$ & 1 & + & Isolated from Salmo gairdneri in Denmark & C.J. Rodgers (University of Tarragona, Spain) \\
\hline $13 / 86^{*}$ & I & + & Isolated from Salmo gairdneri in England & C.J. Rodgers (University of Tarragona, Spain) \\
\hline A100, A102, & 1 & + & Trout-isolated strain & I. Márquez (SERIDA, Spain) \\
\hline $150 / 05,158 / 05,382 / 05$ & I & + & Trout-isolated strain & Proaqua Nutrition S.A. \\
\hline $137 / 76^{*}, 138 / 76^{*}$ & I & + & Trout-isolated strain & C.J. Rodgers (University of Tarragona, Spain) \\
\hline NCTC $10478^{*}$ & I & + & Isolated from S. gairdneri in USA & C.J. Rodgers (University of Tarragona, Spain) \\
\hline NCMB 1315* & NT & - & Isolated from S. gairdneri in USA & C.J. Rodgers (University of Tarragona, Spain) \\
\hline NCMB $1316^{*}$ & I & - & Isolated from S. gairdneri in USA & C.J. Rodgers (University of Tarragona, Spain) \\
\hline Yr.V187/09/115* & 1 & - & Isolated from S. salar in Norway & C.J. Rodgers (University of Tarragona, Spain) \\
\hline Yr.V187/09/700* & $\|$ & - & Trout-isolated strain & C.J. Rodgers (University of Tarragona, Spain) \\
\hline ATCC $29473^{*}$ & 1 & - & Isolated from S. gairdneri in USA & C.J. Rodgers (University of Tarragona, Spain) \\
\hline RD38* & 1 & + & Trout-isolated strain & R.L. Davies, University of Stirling (Scotland) \\
\hline AL 3017 & NT & - & Trout-isolated strain & M.D. Furones, IRTA (Tarragona) \\
\hline \multicolumn{5}{|l|}{ E. coli } \\
\hline DH5a入pir & NT & NT & $\begin{array}{l}\text { F'}^{\prime} / \text { endA1 hsdR17 (rk-mk+) supE44 } \\
\text { thi-1 recA1 gyrA (NalR) } \lambda \text { (pir) }\end{array}$ & [30] \\
\hline S17-1入pir & NT & NT & $\lambda$ (pir) hsdR pro thi, RP4-2 Tc::Mu Km::Tn7 & [31] \\
\hline
\end{tabular}

probe to perform hybridization. For genome location of the mini-Tn5 transposon mutated gene, plasmid was obtained by the Kado and Liu [35] method, and total DNA was extracted from the Y. ruckeri strains 150 and 956 using GenElute Mammalian Genomic DNA Purification Kit-Sigma. DNA extracted from both procedures was separated by agarose gel electrophoresis and after transfer to nylon membranes, submitted to hybridization using as a probe a labelled 455 bp PCR-generated internal fragment

Table 2 Plasmids used in this study

\begin{tabular}{lll}
\hline Plasmid & Characteristics & $\begin{array}{l}\text { Source or } \\
\text { reference }\end{array}$ \\
\hline pGBM5 & Spc'/Smr', lac promoter & {$[32]$} \\
pGBM5-yras & pGBM5 harboring yras gene & This study \\
pUC19 & Apr', cloning vector & Pharmacia \\
pUT mini-Tn5 Km2 & $\begin{array}{l}\text { Apr, oriR6K, mobRP4, tnp, } \\
\text { mini-Tn Km2 (Kmr) }\end{array}$ & {$[33]$} \\
\hline
\end{tabular}

of the $y \mathrm{raS}$ gene. PCR was performed using the following primers: yraS-a (5'-ACCGAAGCGCCAGCAGA-3') and yraS-b (5'-AGTGTCGCTGGATTACC-3').

The acrA mutant was obtained after replica plating of a mini-Tn5 transposon library on TSA medium and TSA containing $0.5 \% \mathrm{w} / \mathrm{v}$ SDS. Colonies able to grow on TSA but unable to do so in the presence of SDS were selected. Unique transposon insertion in the genome of the bacterium was assessed by Southern blot analysis as described above using as a probe the labelled region of the kanamycin gene from the Tn 5 transposon.

To obtain the complete sequence of yraS (the gene responsible for the $\mathrm{HSF}^{+}$phenotype), genomic DNA from the $Y$. ruckeri yraS mutant strain was digested with EcoRI and PstI restriction enzymes to obtain the DNA regions adjacent to the $5^{\prime}$ and $3^{\prime}$ ends, respectively, from the kanamycin gene of the mini-Tn $5 \mathrm{Km} 2$ transposon. The restriction fragments were ligated into the pUC19 plasmid previously digested with the corresponding enzymes and 
dephosphorylated, and the mixture was used to trans-

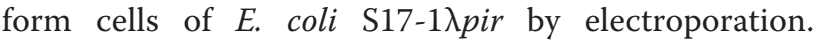
Transformants were selected on $2 \times \mathrm{TY}$ agar medium containing kanamycin and ampicillin. Plasmid DNA was obtained and sequencing was carried out using primers from the mini-Tn5 Km2 transposon sequence (Tn5sec:5'-AAACGCGTATTCAGGCT-3', and Tn5-sec2:5' GCGTATTCAGGCTGACC-3'). Based on the obtained sequences new primers were designed to complete the yraS gene sequence. DNA sequencing was performed by the dideoxy chain termination method with the BigDye Terminator version 3.1 (Applied Biosystems) according to the manufacturer's instructions in an ABI Prism 3730 DNA sequencer.

A similar procedure was performed for the sequencing of the acrA gene. The DNA from the selected mutant was digested with $K p n \mathrm{I}$ and $X b a \mathrm{I}$ restriction enzymes for the sequencing of the DNA region located at $5^{\prime}$ and $3^{\prime}$ position with respect to the kanamycin gene. After DNA digestion, the fragments were ligated into the pUC19 plasmid and the mixture was used to transform cells of

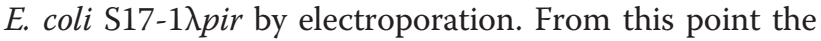
same procedure described in the previous paragraph was followed.

To complement the $Y$. ruckeri yraS mutant and $Y$. ruckeri 956 strains, the yraS gene was amplified from the parental strain by PCR using Biotools DNA polymerase and primers yras-S (5'-CCTGGTCGACGGTTGG TATTGTCTGGT-3') and yras-E (5'-GGCGGAATTC AGTCAGTGAGATAACGA-3'). The SalI and EcoRI restriction sites (in italics) were introduced into the sequences of yras-S and yras-E, respectively, to clone the PCR product digested with SalI and EcoRI into pGBM5 [32] previously digested with the same enzymes. The resulting plasmid was designated pGBM5-yraS (Table 2). Transfer of pGBM5-yraS to 150yraS and 956 strains was carried out by electroporation and colonies were selected on 2xTY agar medium containing streptomycin. The plasmid was recovered from the transformants, digested by SalI and EcoRI and analysed by agarose gel electrophoresis to confirm the presence of the insert.

SDS-PAGE analysis of the HSF presence in Y. ruckeri strains $Y$. ruckeri cultures from the different strains were grown in NB until the early stationary phase of growth. Bacterial cultures were centrifuged at $13,000 \mathrm{rpm}$ for $10 \mathrm{~min}$ and cells re-suspended in $62.5 \mathrm{mM}$ Tris- $\mathrm{HCl}(\mathrm{pH}$ 6.8). Cells were broken by ultrasound treatment in an ice bath, samples were then centrifuged at 13,000 rpm for $30 \mathrm{~min}$ at $4^{\circ} \mathrm{C}$ and the supernatants were used for SDSPAGE electrophoresis. A 1:3 volume of 2x Laemmli sample buffer was added to the samples and then they were subjected to SDS-PAGE in $12 \%$ gels in a cool room overnight at $15 \mathrm{~mA}$. Gels were then incubated at $20^{\circ} \mathrm{C}$ for four hours and finally screened for white opaque bands, a product of the degradation of the SDS contained in the gel, over a dark background. When needed, samples were treated at $100^{\circ} \mathrm{C}$ for $10 \mathrm{~min}$ before SDS-PAGE. Sudan black staining was performed after gel electrophoresis by immersion of the gel in a Sudan Black solution $(0.5 \% \mathrm{w} / \mathrm{v})$ in ethanol. The gels were examined for blue bands after destaining.

\section{PCR detection of the yraS gene in different $Y$. ruckeri strains}

The presence of the yraS gene in a variety of $Y$. ruckeri strains from different origins and geographic areas was examined by PCR using the following primers: yraS-a (5'-ACCGAAGCGCCAGCAGA-3') and yraS-b (5'-AGT GTCGCTGGATTACC-3'). The amplification reaction was performed in a Perkin-Elmer 9700 GeneAmp thermocycler with an initial denaturation cycle at $94^{\circ} \mathrm{C}$ for $5 \mathrm{~min}$, followed by 25 cycles of amplification (denaturation at $94^{\circ} \mathrm{C}$ for $30 \mathrm{~s}$, annealing at $52^{\circ} \mathrm{C}$ for $1 \mathrm{~min}$, and extension at $72^{\circ} \mathrm{C}$ for $1 \mathrm{~min}$ ), and a final 7 -min elongation step at $72^{\circ} \mathrm{C}$. The presence of a 455 bp amplicon was confirmed by agarose gel electrophoresis.

\section{Gas chromatography-mass spectrometry of SDS derivative product and SDS quantification}

Stationary-phase cultures of $Y$. ruckeri parental and yraS mutant strains were used to inoculate (1:100) $250 \mathrm{ml} \mathrm{Er-}$ lenmeyer flasks containing $20 \mathrm{ml}$ of NB supplemented with SDS $(0.25 \% \mathrm{w} / \mathrm{v})$. A similar flask containing NB was used as control. After 24 hours of incubation at $28^{\circ} \mathrm{C}$ and $250 \mathrm{rpm}$, cultures were filtered through disc filters with a pore size of $0.45 \mu \mathrm{m}$ (Pall Life Sciences). The filtrates were dried in a speed vac and re-suspended in $1 \mathrm{ml}$ of chloroform. Samples were then subjected to a gas chromatography-mass spectrometry system consisting of an Agilent model 6890 N-5975B (Santa Clara, California, USA) equipped with a capillary column Agilent $19091 \mathrm{~J}-433 \mathrm{HP}-5(30 \mathrm{~m} \times 0.25 \mathrm{~mm}, 0.25 \mu \mathrm{m}$ film thickness, Agilent Technologies, California, USA). A 1-min splitless injection of $1 \mu \mathrm{l}$ of a 1:400 dilution of each sample was used. Linear velocity of the carrier gas (helium): $36 \mathrm{~cm} / \mathrm{s}(1 \mathrm{ml} / \mathrm{min})$. Oven program: $40^{\circ} \mathrm{C}, 20^{\circ} \mathrm{C} / \mathrm{min}$ to $250^{\circ} \mathrm{C} ; 10^{\circ} \mathrm{C} / \mathrm{min}$ to $300^{\circ} \mathrm{C}$ (hold for $5 \mathrm{~min}$ ). Temperatures of injector, source, interface and quadrupole were $270^{\circ} \mathrm{C}$, $230^{\circ} \mathrm{C}, 280^{\circ} \mathrm{C}$ y $150^{\circ} \mathrm{C}$, respectively. $70 \mathrm{eV}$ was used for ionization. Mass spectra were recorded scanning the 20-550 m/z range.

For SDS quantification, at different times during the growth curve samples were withdrawn and supernatant obtained by centrifugation at $13,000 \mathrm{rpm}$ for $10 \mathrm{~min}$. Samples of $1 \mu \mathrm{l}$ of 1:3 supernatant dilutions were mixed with $200 \mu \mathrm{l}$ Stains-all assay solution [14] and the absorbance was read at $438 \mathrm{~nm}$ using a PowerWave $^{\mathrm{TM}} \mathrm{XS}$ 
Microplate Spectrophotometer (BioTek). New calibration curves $(0-0.1 \% \mathrm{w} / \mathrm{v}$ SDS) were acquired with each set of samples. In all the cases, samples were analysed by triplicate.

\section{MIC determination}

The MIC for different compounds was determined by the dilution method in NB. A bacterial inoculum was prepared by diluting 100 times in NB a 0.5 McFarland suspension $\left(10^{8} \mathrm{CFU} / \mathrm{ml}\right)$ from an exponential-phase culture $\left(\mathrm{OD}_{600}: 0.5\right)$. One $\mathrm{ml}$ of the cell suspension was added to each tube containing the appropriate dilution of the tested compound. Then, cultures were incubated for $24 \mathrm{~h}$ at $28^{\circ} \mathrm{C}$ and $250 \mathrm{rpm}$. Growth inhibition was evaluated macroscopically, except for SDS, which was determined by serial dilutions and plate counts. The $\mathrm{MIC}$ value corresponds to the first dilution of each compound where no growth at all was observed. MIC values were tested in triplicate.

\section{Animal experiments}

Animal experiments were performed in accordance with the European legislation governing animal welfare, and they were authorized and supervised by the Ethics Committee of Universidad de Oviedo. Rainbow trout (Oncorhynchus mykiss) of about 10-15 g obtained from a commercial fish farm were used in all the experiments. Fish were kept in $60 \mathrm{ltanks}$ at $18^{\circ} \mathrm{C} \pm 1$ in dechlorinated water. Each batch was microbiologically analysed for potential pathogens before and during all the experimental process.

For $\mathrm{LD}_{50}$ determination, $Y$. ruckeri parental and yraS mutant strains cultures were grown to an $\mathrm{OD}_{600}$ of 0.5 $0.6\left(\mathrm{OD}_{600} 0.5=10^{8} \mathrm{CFU} / \mathrm{ml}\right)$, harvested by centrifugation and washed twice with PBS. Cells were re-suspended in PBS and logarithmic dilutions were prepared. Groups of 10 fish were challenged by intraperitoneal injection of $100 \mu \mathrm{l}$ of each dilution from $10^{2}$ to $10^{8} \mathrm{CFU} / \mathrm{ml}$. Mortality was monitored daily over a 7-days period, and $\mathrm{LD}_{50}$ was calculated according to the PROBIT method using the SPSS statistical package for Windows, establishing a 95\% confidence limit. $\mathrm{LD}_{50}$ experiments were performed in duplicate. Aliquots of bacterial suspensions injected in each dilution were plated on NA and after incubation for two days at $18^{\circ} \mathrm{C}$ colonies were counted for further $\mathrm{LD}_{50}$ determination. Dead animals were examined bacteriologically to confirm the presence of $Y$. ruckeri. Control fishes were injected with an equal volume of PBS.

\section{In silico analysis}

Sequences were compared to those in the databases with the BLAST (Basic Local Alignment Search Tool) program. Protein sequences were aligned with MUSCLE http://www.ebi.ac.uk/Tools/msa/muscle/ [36] using default parameters. Alignment was manually inspected to correct inaccurately situated residues and a maximum likelihood tree was built with MEGA software http://www. megasoftware.net/ [37], using WAG + G model [38] as most suitable amino acid substitution method and 4 Gamma categories. Bootstrap analysis was performed by resampling 500 times. Topology was checked and edited using iTOL http://itol.embl.de/ [39,40] and manual graphical corrections were performed using Adobe Illustrator CS6 (Adobe Systems, USA).

\section{Availability of supporting data}

The yraS sequence was deposited in GenBank under accession number KF421132.

\section{Additional files}

\begin{abstract}
Additional file 1: Figure S1. GC-MS spectra of the components found in the culture supernatant after incubation of $Y$. ruckeri. Y. ruckeri parental and yraS mutant strains were grown for $24 \mathrm{~h}$ at $28^{\circ} \mathrm{C}$ in NB containing $0.25 \%$ SDS. After $24 \mathrm{~h}$ of incubation at $28^{\circ} \mathrm{C}$, cultures were filtrated. Filtrates were dried by vacuum centrifuge and finally resuspended in chloroform. Samples of $1 \mu$ of 1:400 dilutions were then analysed by GC-MS; framing the peak amplified in graph E. (A) Commercial 1-dodecanol (control). (B) Sample from Y. ruckeri 150 culture. (C) Sample from Y. ruckeri yraS mutant culture. (D) Sample from culture medium without inoculation. (E) Magnification of the peak relative to the retention time corresponding to 1-dodecanol from the sample of the $Y$. ruckeri parental strain culture.
\end{abstract}

Additional file 2: Figure S2. Phylogenetic tree based on the deduced amino acid sequence of the YraS protein from Y. ruckeri. The protein alignment was carried out by the MUSCLE program [14], and further corrected manually and a phylogenetic tree of maximum likelihood was constructed using the MEGA program [36]. The topology was edited with the iTOL program [38,39].

Additional file 3: Figure S3. SDS-PAGE of cell extracts of different $Y$. ruckeri strains after Sudan black staining. Electrophoresis was performed at $15 \mathrm{~mA}$ in a cool room for $16 \mathrm{~h}$. Then, the gel was incubated at $20^{\circ} \mathrm{C}$ for $4 \mathrm{~h}$ and stained with Sudan black dye. Cell extract from: lane 1,

Y. ruckeri parental strain; lane 2, yraS; lane 3, yraS ${ }^{+}$. Molecular masses are indicated in $\mathrm{kDa}$ on the left side of the gel. Only strains bearing the yras gene were positive for the presence of the $120 \mathrm{kDa}$ band stained with Sudan black

Additional file 4: Figure S4. Mean cumulative percent mortality of rainbow trout following challenge with Y. ruckeri parental and yraS mutant strains. Single groups of 10 fish were challenged by intraperitoneal infection with $1.8 \times 10^{2}(\mathbf{a}), 3.5 \times 10^{2}(\mathbf{(}), 1.8 \times 10^{4}(\boldsymbol{\Delta})$ and $3.5 \times 10^{4}(\mathbf{A})$ CFU of parental (continuous line) and yraS mutant (dotted line) strains, and mortality was monitored every day.

Additional file 5: Figure S5. Effect of glucose on the $\mathrm{HSF}^{+}$phenotype of $Y$. ruckeri. Plates containing M9C with $0.5 \% \mathrm{~W} / \mathrm{V}$ SDS (A); and the same medium plus $1 \% \mathrm{w} / \mathrm{v}$ of glucose (B) were spotted with $5 \mu \mathrm{l}$ of early stationary phase cultures of $Y$. ruckeri parental and yraS strains. After 96 h of incubation at $28^{\circ} \mathrm{C}$, plates were photographed. The $\mathrm{HSF}^{+}$phenotype, indicated by the creamy white colony corresponding to the parental strain grown in the absence of glucose (A), was converted to $\mathrm{HSF}^{-}$in the presence of glucose (B).

\section{Competing interest}

The authors declare that they have no competing interests.

\section{Authors' contributions}

JAG, RN and JM designed the study and wrote the manuscript. RN, JM and DC performed mutant's selection, genetic manipulations and virulence 
testing. RN, JM, PR and JAG analysed the data. All authors read and approved the final manuscript.

\section{Authors' information}

This work was carried out in the Área de Microbiología, Departamento de Biología Funcional, Facultad de Medicina, IUBA, Universidad de Oviedo, 33006 Oviedo, Asturias, Spain. RN, JM and PR are Doctors; DC is PhD student; and JAG is Professor of Microbiology.

\section{Acknowledgments}

The authors thanks to Dr. Dolors Furones for her generously providing $Y$. ruckeri strains and help during the development of this work. Roberto Navais and Desirée Cascales were the recipients of grants from the Spanish Ministerio de Ciencia e Innovación (MICINN). The study was supported by the MINECO project AGL2012-35808 and FEDER fund.

Received: 26 May 2014 Accepted: 12 August 2014

Published online: 30 September 2014

\section{References}

1. Romalde $J$, Magariños B, Barja $J$, Toranzo AE: Antigenic and molecular characterization of Yersinia ruckeri. Proposal for a new intraspecific classification. Syst Appl Microbiol 1993, 16:411-419.

2. Davies RL, Frerichs GN: Morphological and biochemical differences among isolates of Yersinia ruckeri obtained from wide geographical areas. J Fish Dis 1989, 12:357-365.

3. Tobback E, Decostere A, Hermans K, Haesebrouck F, Chiers K: Yersinia ruckeri infections in salmonid fish. J Fish Dis 2007, 30:257-268.

4. Fernández L, Méndez J, Guijarro JA: Molecular virulence mechanisms of the fish pathogen Yersinia ruckeri. Vet Microbiol 2007, 125:1-10

5. Dahiya I, Stevenson RMW: The ZnuABC operon is important for Yersinia ruckeri infections of rainbow trout, Oncorhynchus mykiss (Walbaum). J Fish Dis 2010, 33:331-340.

6. Dahiya I, Stevenson RMW: The UvrY response regulator of the BarA-UvrY two-component system contributes to Yersinia ruckeri infection of rainbow trout (Oncorhynchus mykiss). Arch Microbiol 2010, 192:541-547.

7. Méndez J, Fernández L, Menéndez A, Reimundo P, Pérez-Pascual D, Navais R, Guijarro JA: A chromosomally located traHIJKCLMN operon encoding a putative type IV secretion system is involved in the virulence of Yersinia ruckeri. Appl Environ Microbiol 2009, 75:937-945.

8. Méndez J, Reimundo P, Pérez-Pascual D, Navais R, Gómez E, Guijarro JA: A novel $c d s A B$ operon is involved in the uptake of $L$-cysteine and participates in the pathogenesis of Yersinia ruckeri. J Bacteriol 2011, 193:944-951.

9. Furones MD, Gilpin MJ, Alderman DJ, Munn CB: Virulence of Yersinia ruckeri serotype I strains is associated with a heat sensitive factor (HSF) in cell extracts. FEMS Microbiol Lett 1990, 66:339-344.

10. Furones MD, Gilpin ML, Munn CB: Culture media for the differentiation of isolated of Yersinia ruckeri, based on detection of a virulence factor. J Appl Bacteriol 1993, 74:360-366.

11. Hagelueken $G$, Adams TM, Wiehlmann L, Widow U, Kolmar H, Tümmler B, Heinz DW, Schubert W-D: The crystal structure of SdsA1, an alkylsulfatase from Pseudomonas aeruginosa, defines a third class of sulfatases. Proc Natl Acad Sci U S A 2006, 103:7631-7636.

12. Daiyasu $H$, Osaka $K$, Ishino $Y$, Toh $H$ : Expansion of the zinc metallohydrolase family of the beta-lactamase fold. FEBS Lett 2001, 503:1-6.

13. Chaturvedi $V$, Kumar $A$ : Bacterial utilization of sodium dodecyl sulfate. Int J Appl Biol Pharmaceut Tech 2010, 1:1126-1131.

14. Rusconi F, Valton E, Nguyen R, Dufourc E: Quantification of sodium dodecyl sulfate in microliter-volume biochemical samples by visible light spectroscopy. Anal Biochem 2001, 295:31-37.

15. Rosenberg EY, Ma D, Nikaido H: AcrD of Escherichia coli is an aminoglycoside efflux pump. J Bacteriol 2000, 182:1754-1756.

16. Baucheron S: AcrAB-Tolc directs effluxmediated multidrug resistance in Salmonella enterica serovar Typhimurium DT104. Antmicrob Agents Chemother 2004, 48:3729-3735.

17. Bavro VN: Assembly and channel opening in a bacterial drug efflux machine. Mol Cell 2008, 11:114-121.
18. Lillis $\mathrm{V}$, Dodgson KS, White FF: Initiation of activation of a pre-mergent herbicide by novel alkyl sulfatase of Pseudomonas putida FLA. Appl Environ Microbiol 1983, 46:988-994.

19. Bateman TJ, Dodgson KS, White GF: Primary alkyl sulfatase activities of the detergent-degrading bacterium Pseudomonas C12B. Biochem J 1986 236:401-408.

20. Matcham GJW, Bartholomev B, Dodgson KS, Payne WJ, Fitzgerald W: Stereospecificity and complexity of microbial sulfohydrolases involved in the biodegradation of secondary alkyl sulfate detergents. FEMS Microbiol Lett 1977, 1:197-200.

21. Boltes I, Czapinska H, Kahnert A, von Bülow R, Dierks T, Schmidt B, von Figura K, Kertesz MA, Uson I: 1.3 A structure of arylsulfatase from Pseudomonas aeruginosa established the catalitic mechanism of sulfate ester cleavage in the sulfatase family. Structure 2001, 9:483-491.

22. Müller I, Kahnert A, Pape T, Sheldrick GM, Meyer-Klauche W, Dierks T, Kerrtesz M, Uson I: Cristal structure of the alkylsulfatase AtsK: Insights into the catalytic mechanism of the Fe (II) alphaketoglutarate-dependent dioxygenase superfamily. Biochemistry 2004, 43:3075-3088.

23. Hanson SR, Best MD, Wong C-H: Sulfatases: structure, mechanism, biological activity, inhibition, and synthetic utility. Angew Chem Int Ed 2004, 43:5736-5763.

24. Tazuke Y, Matsuda K, Adachi K, Tsukada Y: Purification and properties of bile acid sulfate sulfatase from Pseudomonas testosteroni. Biosci Biotechnol Biochem 1994, 58:889-894.

25. Osteras M, Boncompagni E, Vincent N, Poggi MC, LeRudulier D: Presence of a gene encoding choline sulfatase in Sinorhizobium meliloti bet operon: Choline-o-sulfatase is metabolized into glycine betaine. Proc Natl Acad Sci U S A 1998, 5:11394-11399.

26. Chaturvedi V, Kumar A: Isolation of a strain of Pseudomonas putida capable of metabolizing anionic detergents sodium docecyl sulfate (SDS). Iran J Microbiol 2011, 1:47-53.

27. Hosseini F, Malekzadeh F, Amirmozafari N, Ghaemi N: Biodegradation of anionic surfactants by isolation bacteria from activated sludge. Int $J$ Environ Sci Tech 2007, 4:1237-1242.

28. Shukor MY, Husin WSW, Rahman MFA, Shamaan NA, Syed MA: Isolation and characterization of an SDS-degrading Klebsiella oxytoca. J Environ Biol 2009, 30:129-134.

29. Romalde $\mathrm{J}$, Conchas RF, Toranzo AE: Evidence that Yersinia ruckeri possesses a high affinity iron uptake system. FEMS Microbiol Lett 1991, 64:121-125.

30. Woodcock DM, Crowther PJ, Doherty J, Jefferson S, DeCruz E, Noyer-Weidner M, Smith SS, Michael MZ, Grahan MW: Quantitative evaluation of Escherichia coli host strains for tolerance to cytosine methylation in plasmid and phage recombinants. Nucleic Acids Res 1989, 17:3469-3478.

31. Simon $R$, Priefer $U$, Pühler $A$ : A broad host range mobilization system for in vivo genetic engineering: transposon mutagenesis in gram negative bacteria. Nat Biotechnol 1983, 1:784-791.

32. Manen D, Pougeon M, Danmay P, Geiselmenn J: A sensitive reporter gene system using bacterial luciferase based on a series of plasmid cloning vectors compatible with derivatives of pBR322. Gene 1997 28:197-200.

33. de Lorenzo V, Herrero M, Jakubzik U, Timmis KN: Mini-Tn5 Transposon derivatives for insertion mutagenesis, promoter probing, and chromosomal insertion of cloned DNA in Gram-negative eubacteria. J Bacteriol 1990, 172:6568-6572.

34. Fernández $L$, Prieto $M$, Guijarro JA: The iron- and temperature-regulated haemolysin YhIA is a virulence factor of Yersinia ruckeri. Microbiology 2007, 153:483-489.

35. Kado Cl, Liu ST: Rapid procedure for detection and isolation of large and small plasmids. J Bacteriol 1981, 145:1365-1373.

36. Edgar RC: MUSCLE: multiple sequence alignment with high accuracy and high throughput. Nucleic Acids Res 2004, 32:1792-1797.

37. Tamura K, Peterson D, Peterson N, Stecher G, Nei M, Kumar S: MEGA5: molecular evolutionary genetics analysis using maximum likelihood, evolutionary distance, and maximum parsimony methods. Mol Biol Evol 2011, 28:2731-2739.

38. Whelan S, Goldman N: A general empirical model of protein evolution derived from multiple protein families using a maximum-likelihood approach. Mol Biol Evol 2001, 18:691-699. 
39. Letunic I, Bork P: Interactive Tree Of Life (iTOL): an online tool for phylogenetic tree display and annotation. Bioinformatics 2007 23:127-128

40. Letunic I, Bork P: Interactive Tree Of Life v2: online annotation and display of phylogenetic tress made easy. Nucleic Acid Res 2011, 39:475-478

doi:10.1186/s12866-014-0221-7

Cite this article as: Navais et al: The heat sensitive factor (HSF) of Yersinia ruckeri is produced by an alkyl sulphatase involved in sodium dodecyl sulphate (SDS) degradation but not in virulence. BMC Microbiology 2014 14:221.

\section{Submit your next manuscript to BioMed Central and take full advantage of:}

- Convenient online submission

- Thorough peer review

- No space constraints or color figure charges

- Immediate publication on acceptance

- Inclusion in PubMed, CAS, Scopus and Google Scholar

- Research which is freely available for redistribution 